\title{
Stairstepping on PWM Inverters Explained
}

\author{
J. Knockaert ${ }^{1 \& 2}$, J. Peuteman ${ }^{2}$, J. Catrysse ${ }^{2}$, R. Belmans ${ }^{1}$ \\ (1) KATHOLIEKE UNIVERSITEIT LEUVEN, DEPARTEMENT ELECTRICAL ENGINEERING (ESAT), \\ ONDERZOEKSGROEP ELECTA \\ Kasteelpark Arenberg 10, B-3001 Heverlee - Belgium \\ Tel. $+32-(0) 16 / 321020$ \\ (2) KATHOLIEKE HOGESCHOOL BRUGGE OOSTENDE, DEPARTEMENT IW\&T \\ Zeedijk 101, B-8400 Oostende - Belgium \\ Tel. $+32-(0) 59$ / 569000 \\ email: jos.knockaert@khbo.be
}

Keywords: Inverter, PWM, stairstepping, DTG

\begin{abstract}
When supplying an induction motor by a PWM-inverter, reflection phenomena occur if the supply is done through long motor cables. Several papers discuss by simulations or experiments the overvoltage appearing at the motor terminals. The consequences of the overvoltage are a widespread topic. This paper deals with other reflection phenomena observed with different PWM-inverters. Stairstepping and the influence of the dead-time generator on the reflections are analysed.
\end{abstract}

\section{Introduction}

When supplying an induction motor by a PWM-inverter, reflection phenomena occur if the supply is done through long motor cables. Several papers discuss by simulations or experiments the overvoltage appearing at the motor terminals $[1,4]$. The consequences of the overvoltage are a widespread topic [5]. This paper deals with other reflection phenomena observed with different PWM-inverters supplying an induction motor through long motor cables.

In the first part, stair stepping is discussed. Stair stepping is a well known phenomenon in high speed digital design. In this paper, it is shown that stair stepping also occurs in PWMinverter fed induction motors. The origin of the phenomenon is totally different. It occurs when the current at the moment of switching is small, typical smaller than $10 \mathrm{~A}$. In small inverters this occurs during almost the whole period, while in large inverters it is hardly seen. As will be proven, it depends on the characteristic impedance of the cable and the dead time. These phenomena only occur when the current commutates from the IGBT to the diode of the inverter bridge. A consequence of the reflection is not an overvoltage, but a delay of the turn on of the diode, due to the multiple reflections. The theory is confirmed by simulations and measurements.

In the second part, the influence of the DTG (dead time generator) on the reflections is discussed. By the DTG the reflection coefficient at inverter side changes, causing additional reflections. At that moment, a second wave is travelling along the motor cable. The DTG can cause large current peaks, which depend on the initial current and the cable impedance. For small inverters, the latter can cause large peaks in comparison to the rated current, i.e. up to twice the rated current and more.

The peak current is calculated in the third part. Another consequence is that the IGBTs are switching at moments normally diodes should be conducting. All of this is proven by simulations and confirmed by measurements.

\section{The Stair Stepping Phenomenon}

\subsection{Measurement}

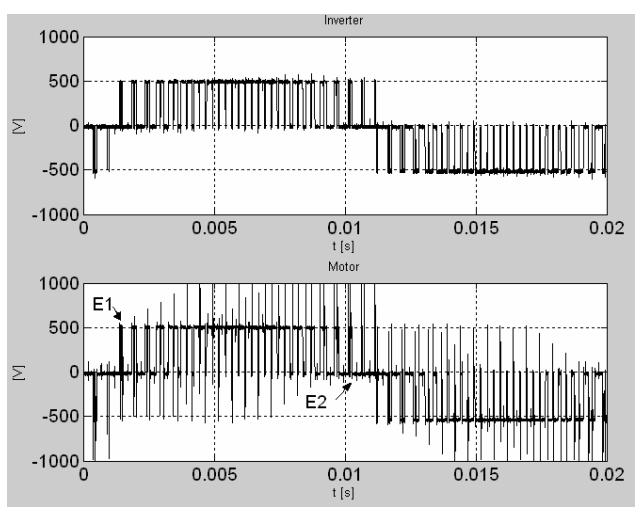

Fig. 1. Voltage at inverter (top) and motor side (bottom) at small load

Measuring at the motor terminals, normally reflections above 1 per unit busvoltage (1 p.u.) occur at turn on. At turn off an equal but negative transient appears. This phenomenon originates due to the impedance mismatch between cable and induction motor. The voltage at the motor terminals nearly doubles. 
Fig. 1 shows a measurement with an inverter connected to a motor by using a long cable. This measurement leads to two important observations. First, not all rising and falling edges result in an overvoltage, as marked by the points E1 and E2 on fig. 1. Second, the reflections at the rising edge do not occur at the beginning of the positive voltage alternation, neither at the falling edge at the end of the positive alternation.

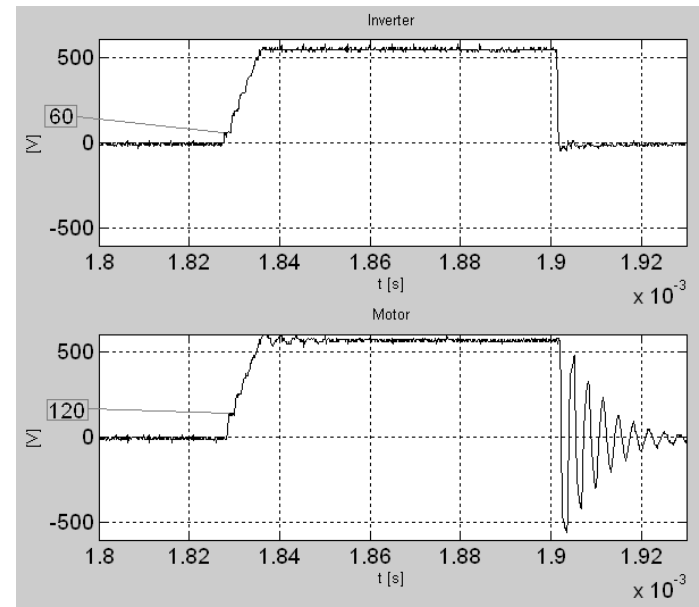

Fig. 2. Zoom of pulse E1; inverter (top) and motor side (bottom)

On fig. 2 (bottom) a zoom of the pulse indicated as E1 on fig. 1 is shown. At the rising edge, the amplitude of the first step is $120 \mathrm{~V}$ or 0.22 p.u. (with 1 p.u. equal to the bus voltage $V_{d c}$ ). As this is already a doubled voltage, due to the high motor impedance for pulses, the output voltage of the inverter should be half of the motor voltage. A measurement at the inverter side confirms this (fig. 2 top). The step voltage starts at the inverter and reflects at the motor. After multiple reflections, the voltage finally reaches a 1 p.u. value, without overvoltage. At the falling edge, a normal overvoltage reflection appears.

\subsection{Explanation}

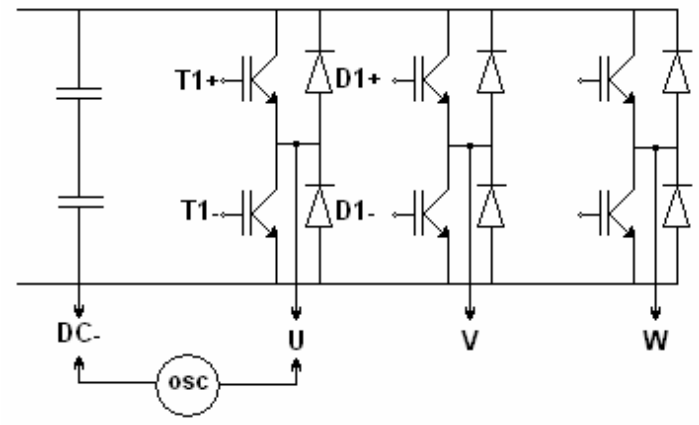

Fig. 3. Measurement between phase $U$ and the DC negative pole

An explanation of the phenomenon has been given with additional measurements as proven in [2]. The cause of stair stepping is summarized here.
Consider the three phase inverter on fig. 3. To explain the phenomenon, only one leg has to be considered. The voltage is measured between one phase and the negative pole of the DC-bus.

Stair stepping is fully described in papers discussing high speed digital design [6]. Stair stepping occurs when the impedance $Z_{c}$ is small in comparison to the load and source impedance. Further investigation shows this is not the case. Measuring the current gives a totally different view on the origin of the steps.

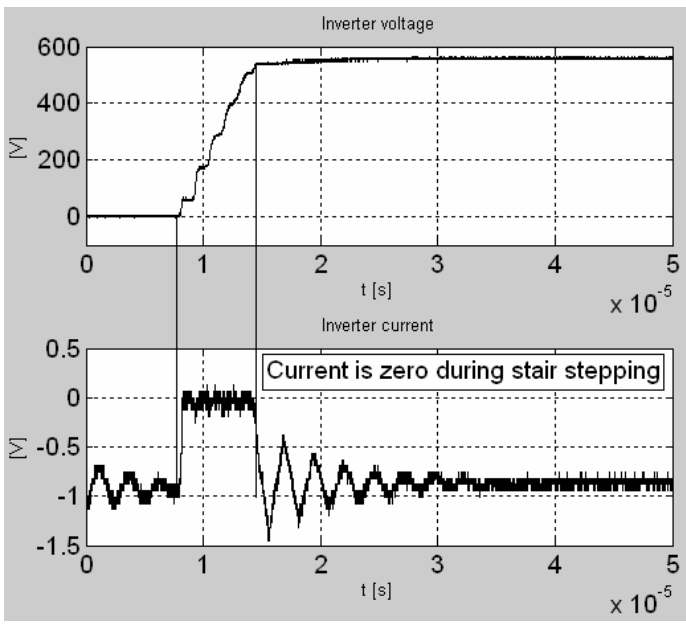

Fig. 4. Stair stepping when the current is small. Commutation from T1- to D1+.

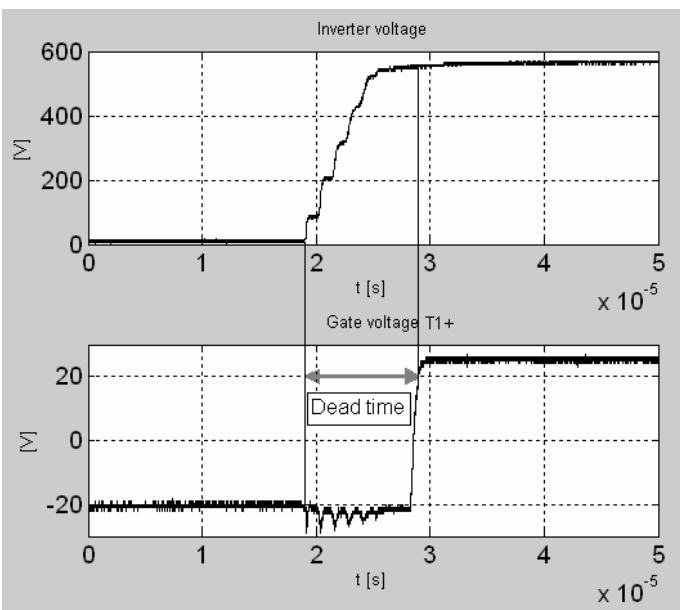

Fig. 5. Diode turns on after several reflections. At that time, the gate of the top IGBT T1+ is still off, due to the dead time.

Essential to understand is that the inverter is not loaded by a motor at the moment of voltage changes, but by the characteristic impedance of the cable. Fig. 4 shows both measured current and the voltage at the inverter output when IGBT T1- turns off. The current should flow through the diode D1+. However, this diode can not turn on, because the anode voltage is less than the cathode voltage. The current becomes zero and remains so, as it is unable to reverse. The 
diode D1- can't conduct as the cathode voltage is larger than the anode voltage. The IGBT T1+ can't conduct, as the dead time is not over yet (fig. 5). Summarized, when T1- turns off, the current falls to zero, i.e. a current step of $\Delta \mathrm{I}$ is added to the initial current $-\Delta \mathrm{I}$. Notice that $\Delta \mathrm{I}$ is in the discussion always the absolute value of the current step. This current wave propagates towards the motor, reflects, returns to the inverter and reflects again as long as $\mathrm{T} 1+$ or $\mathrm{D} 1+$ do not conduct. At each reflection the current remains zero at the inverter terminals and equals the initial value at motor side.

The current wave also starts a voltage wave $\mathrm{Z}_{\mathrm{c}} \cdot \Delta \mathrm{I}$. The reflection factor for voltages at the inverter $K_{\mathrm{iv}}$ is +1 as no component conducts, while the reflection factor $\mathrm{K}_{\mathrm{mv}}$ at the motor is near +1 . In the ideal case of a lossless line, the voltage increases with $\mathrm{Z}_{\mathrm{c}} \cdot \Delta \mathrm{I}$ at each reflection at motor and inverter side, resulting in the stair stepping phenomenon at both inverter and motor side. The reflection lasts until a sufficient voltage (DC-bus voltage $\mathrm{U}_{\mathrm{DC}}+0.7 \mathrm{~V}$ ) is reached or until the dead time of the IGBT is passed. When the diode finally turns on, the current becomes negative again. Despite the inductive nature of the motor, the current changes very fast at the inverter output. The inverter does not see the inductive load but only the characteristic impedance of the cable.

At commutation from IGBT to diode (T1+ to D1- or T1- to $\mathrm{D} 1+)$, there are more, but smaller steps. This results in a small or even no overvoltage at the motor, as was observed on fig. 1.

Bewley diagrams are given in [3] to prove this theory. There it is shown that the current can indeed be zero at the inverter and stay constant at the motor.

To support the previously mentioned physical explanation, a simplified pSpice-model was build [3].

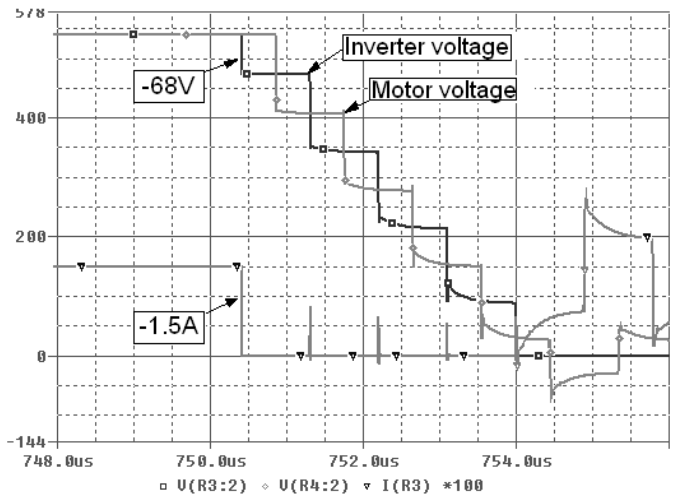

Fig. 6. Simulation of stairs stepping $\left(Z_{c}\right.$ cable $45 \Omega$, current step -1.5 A, voltage step - $68 \mathrm{~V}$, dead time $10 \mu \mathrm{s}$ ). (Current multiplied with 100)

Fig. 6 shows the simulation results of a shielded cable. At turning off the IGBT, the current falls to zero. The current step is -1.5 A. Multiplied by the characteristic cable impedance of $45 \Omega$, this gives a voltage step of $-68 \mathrm{~V}$. After multiple reflections, the diode turns on. This gives a new transient, explained in the next part.

\section{Influence of the DTG}

\subsection{Simulations}

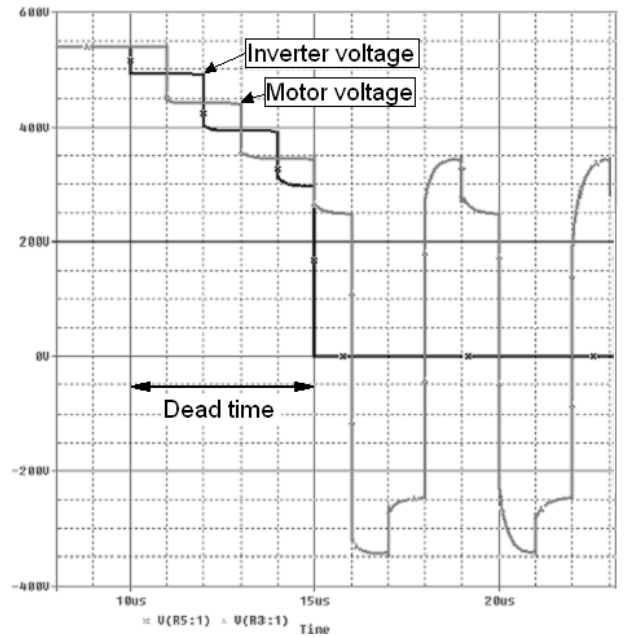

Fig. 7a. Influence of the DTG. Voltage at inverter and motor terminals.

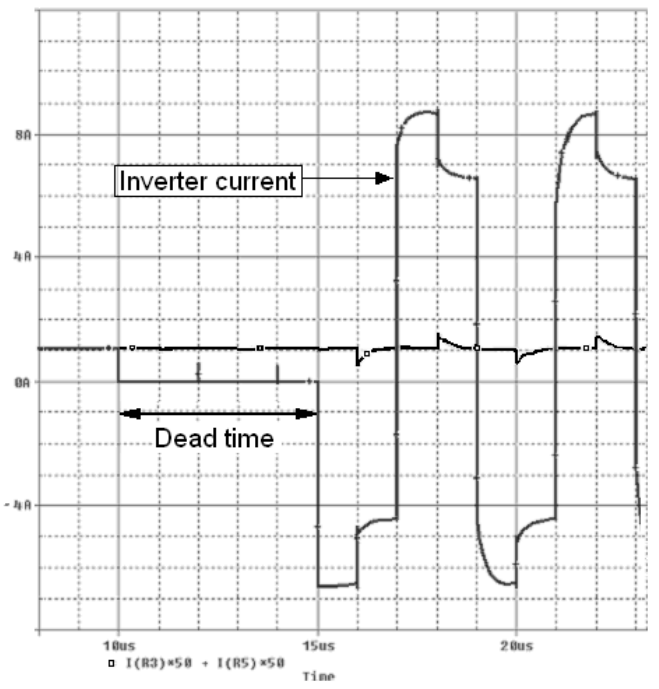

Fig. 7b. Influence of the DTG. Current at inverter and motor terminals.

The additional reflections, occurring when the dead time has passed are interesting. When the dead time is over, the complementary IGBT turns on. If the parallel diode already conducts, nothing changes and the phenomenon of section 3 applies. However, when the stair stepping phenomenon is still bouncing and the dead time has passed, the IGBT pulls the voltage immediately towards $0 \mathrm{~V}$ (IGBT T1-) or $\mathrm{U}_{\mathrm{DC}}$ (IGBT $\mathrm{T} 1+)$. This creates another voltage step, causing another current step. Figs. $7 \mathrm{a}$ and $7 \mathrm{~b}$ show a simulation of these 
reflections. Figs. $8 \mathrm{a}$ and $8 \mathrm{~b}$ show a Bewley diagram explaining the reflections. For easy interpretation the simulation is done with a lossless cable.

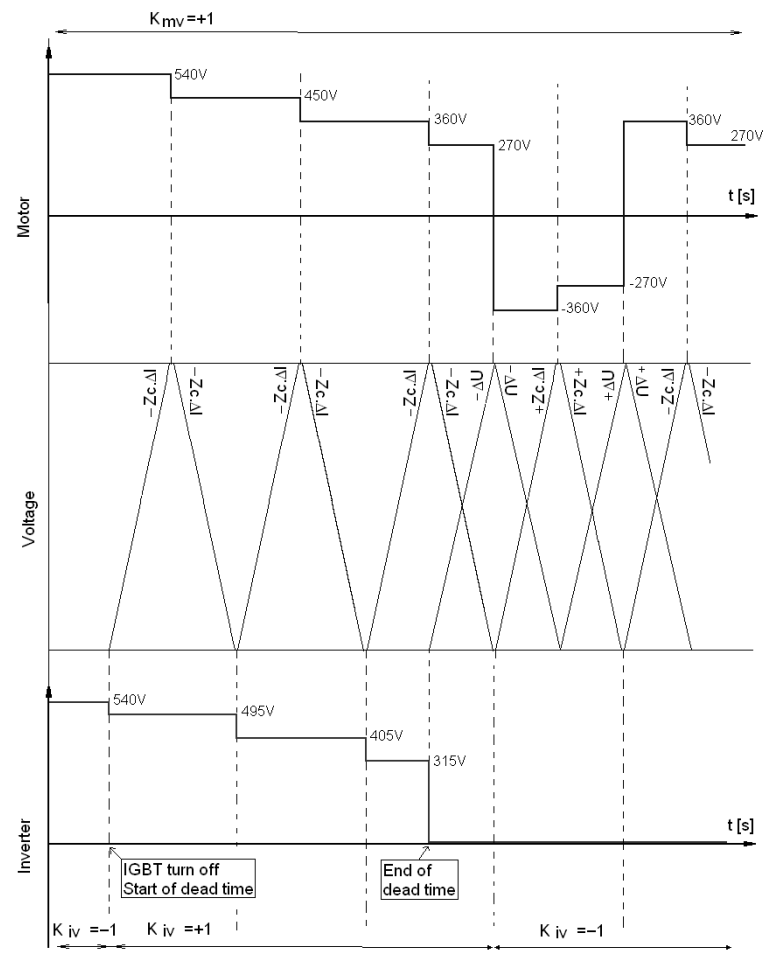

Fig. 8a. Bewley diagram (voltage) explaining transmission line effects caused by DTG.

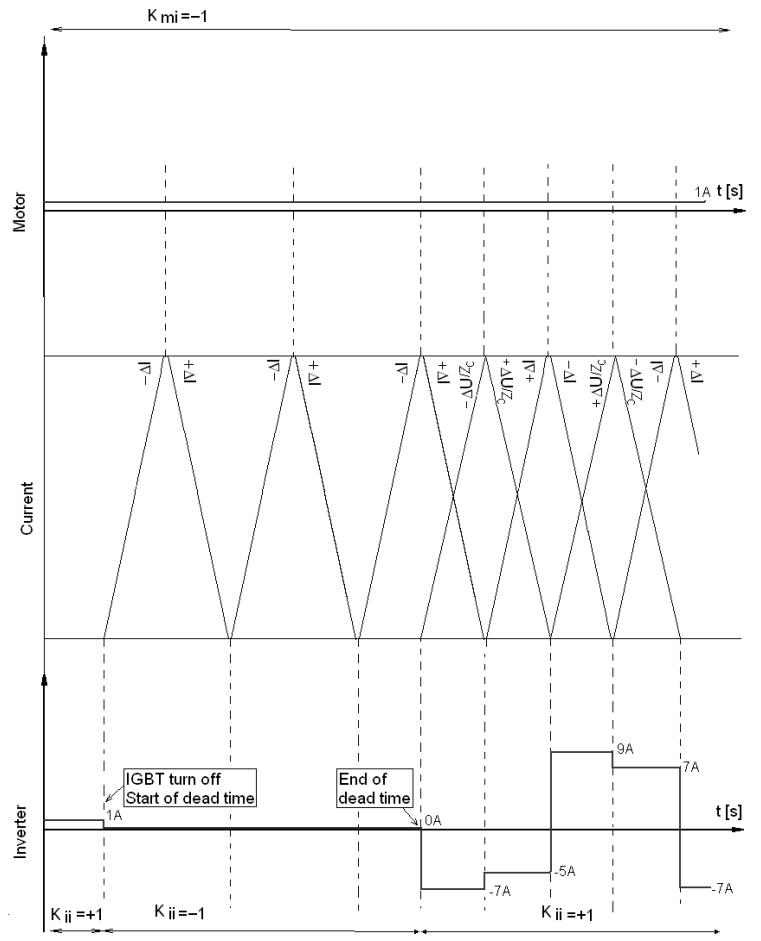

Fig. 8b. Bewley diagram (current) explaining transmission line effects caused by DTG.
The Bewley diagrams on figs. $8 \mathrm{a}$ and $8 \mathrm{~b}$ explain figs. $7 \mathrm{a}$ and 7b. The diagram shows stair stepping, with small steps of 45 V. After some reflections, the dead time is over. T1- turns on, pulling the voltage to $0 \mathrm{~V}$. This involves a voltage step $-\Delta \mathrm{U}$ of $-315 \mathrm{~V}$, generating a current step of $-\Delta \mathrm{U} / \mathrm{Z}_{\mathrm{c}}$. The reflection coefficients at the inverter side change at that instant. There are now two waves travelling, leading to the simulated results.

Interesting to note is that at the instant the dead time is over, the IGBT conducts, while normally the diode should do so. Before turning off $\mathrm{T} 1+$, the current is positive. After turning off, the current falls back to zero while stair stepping occurs. After the dead time, normally diode D1- should be conducting, as the current is normally positive. However, the current oscillates between T1- and D1-.

As can be seen on fig. $8 \mathrm{~b}$, the peak current that can be reached is not the first peak, which is equal to $-\Delta U / Z_{c}$, but the second opposite one, which is $\Delta \mathrm{U} / \mathrm{Z}_{\mathrm{c}}+2 . \Delta \mathrm{I}$. In reality, this peak is always smaller, due to cable losses. In our example, the current before the transient is $1 \mathrm{~A}$, the voltage step of 315 $\mathrm{V}$ causes a current transient of $-7 \mathrm{~A}$. The maximum peak current is then $9 \mathrm{~A}$ as seen on the simulation. The first peak current of $-7 \mathrm{~A}$, is conducted by the IGBT. The second peak current of $9 \mathrm{~A}$ is conducted by the diode, but appears after the current step has travelled already twice through the whole cable. Due to attenuation this $9 \mathrm{~A}$ is always smaller. Nevertheless, the peaks can still be several times the initial current.

As this is only a simulation, with a lossless line, a final prove is measering a peak current of more than twice the initial current.

\subsection{Measurements}

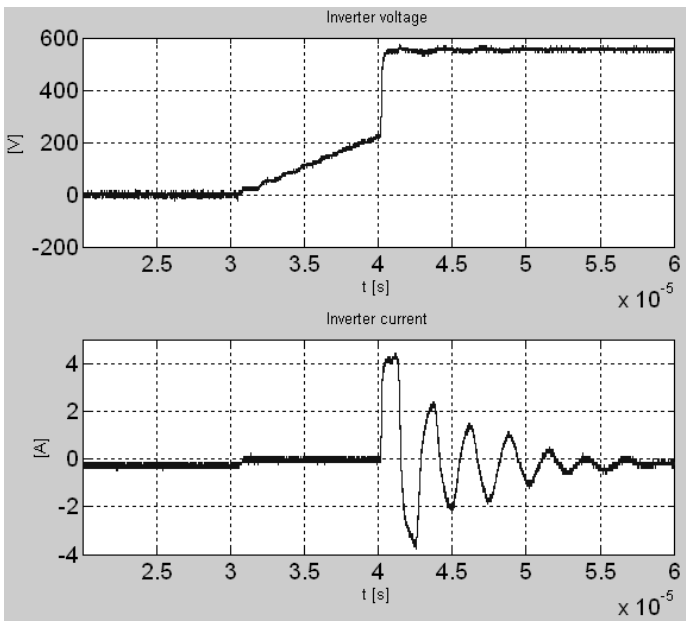

Fig. 9. Interference of the dead time, resulting in a large current step

Fig. 9 shows the discussed phenomenon. The initial current is $-0,25 \mathrm{~A}$. The current falls back to $0 \mathrm{~A}$, giving a current step $\Delta \mathrm{I}$ of $0.25 \mathrm{~A}$ and causing voltage steps $\mathrm{Z}_{\mathrm{c}} \cdot \Delta \mathrm{I}$ of $18 \mathrm{~V}$. The 
voltage rises in several steps up to $225 \mathrm{~V}$. Due to attenuation and distortion, the number of steps are not easy to count. At that moment the dead time has passed and the IGBT T1+ pulls the voltage to $U_{D C}(545 \mathrm{~V})$, causing a step $\Delta U$ of $320 \mathrm{~V}$. The positive peak current for a lossless line is $\Delta \mathrm{U} / \mathrm{Z}_{\mathrm{c}}=$ $320 \mathrm{~V} / 70 \Omega=4,57 \mathrm{~A}$. The negative peak current is $-\Delta \mathrm{U} / \mathrm{Z}_{\mathrm{c}}$ $2 . \Delta \mathrm{I}=-320 \mathrm{~V} / 70 \Omega-(2.0,25 \mathrm{~A})=-5.07 \mathrm{~A}$. The measured positive peak current is 4,2 $\mathrm{A}$ and the negative peak is $-3,6 \mathrm{~A}$, which is smaller due to the attenuation.

\section{Peak value of the current}

Up to this point, only the commutation from IGBT to diode has been discussed, as this is the only moment stair stepping can occur. On the commutation from diode to IGBT , there is also a current peak (fig. 10). The commutation is from diode D1- to an IGBT T1+. This transition always happens without stair stepping. The voltage step $\Delta \mathrm{U}$ is $\mathrm{U}_{\mathrm{DC}}$, which is $555 \mathrm{~V}$ in this case. The current step is then $\mathrm{U}_{\mathrm{DC}} / \mathrm{Z}_{\mathrm{c}}$. This step is added to the initial current. The largest current step at commutation from diode to IGBT is $\Delta \mathrm{I}_{\mathrm{d} 2 \mathrm{i} \_\max }=\mathrm{I}+\mathrm{U}_{\mathrm{DC}} / \mathrm{Z}_{\mathrm{c}}$. On fig. 10, this is in theory $0,65 \mathrm{~A}+555 \mathrm{~V} / 70 \Omega=8,57 \mathrm{~A}$. The measured value is $8,4 \mathrm{~A}$.

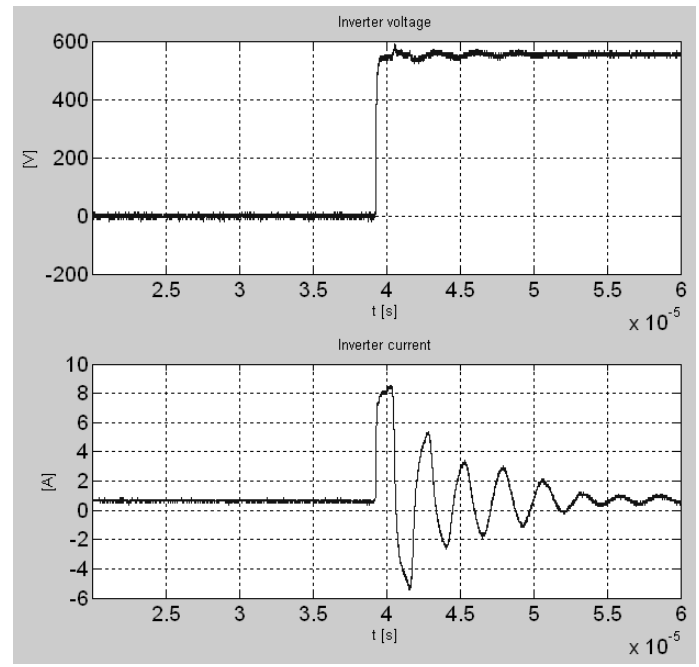

Fig. 10. Largest possible current step

The question that arises is, what is the worst possible case? Theory and practice provide a different answer. At commutation from diode to IGBT, the peak current is $\Delta \mathrm{I}_{\mathrm{d} 2 \mathrm{i} \text { max }}$ $=\mathrm{I}+\mathrm{U}_{\mathrm{DC}} / \mathrm{Z}_{\mathrm{c}}$. On commutation from IGBT to diode, the peak current is $\Delta \mathrm{I}_{\mathrm{i} 2 \mathrm{~d} \_ \text {max }}=2 . \Delta \mathrm{I}+\Delta \mathrm{U} / \mathrm{Z}_{\mathrm{c}}$, where $\Delta \mathrm{I}$ is in fact I. The current from IGBT to diode is larger than the current from diode to IGBT on the following condition:

$$
\begin{aligned}
& \Delta I_{i 2 d_{-} \max }>\Delta I_{d 2 i_{-} \max } \\
& 2 . I+\frac{\Delta U}{Z_{C}}>I+\frac{U_{D C}}{Z_{C}} \\
& \Delta U>U_{D C}-I . Z_{c}
\end{aligned}
$$

This can never happen. The largest $\Delta \mathrm{U}$ occurs, when the dead time is so small, the wave can't travel through the cable and back. In that case, only one step occurs. So the maximum voltage step at commutation from IGBT to diode is in fact $\mathrm{U}_{\mathrm{dc}}-\mathrm{I}_{\mathrm{c}}$. This means, the largest current peaks are in theory equal at commutation from IGBT to diode or vice versa. At switching from diode to IGBT this happens always. At switching from IGBT to diode, the worst case appears when the dead time is smaller than twice the propagation time on the cable.

In theory, the peak values can be similar. In practice $\Delta \mathrm{I}_{\mathrm{i} 2 \mathrm{~d} \_ \text {max }}$ is always smaller than $\Delta \mathrm{I}_{\mathrm{d} 2 \mathrm{i} \_\max }$, because the maximum value only occurs at the second reflection (fig. 8b). Due to cable losses, this second reflection is always smaller.

It can be concluded that the largest current is $\mathrm{I}+\mathrm{U}_{\mathrm{DC}} / \mathrm{Z}_{\mathrm{c}}$. For small inverters this can be several times the nominal current.

\section{Conclusions}

Two phenomena were discussed, stair stepping and the current peaks due to the dead time generator. It was proven by simulations, Bewley diagrams and measurements that an inverter, loaded by a transmission line and an induction motor can show some particular phenomena. Stair stepping is especially seen on inverters for smaller currents. Interference of the dead time can cause unexpected current peaks. These current peaks depend on the voltage step and cable impedance superposed on the initial current. For small inverters loaded by a shielded cable, these peaks can be over several times the initial current and can even be several times the rated current.

\section{References}

[1] R. J. Kerkman, D. Leggate, and G. L. Skibinski, "Interaction of drive modulation and cable parameters on AC motor transients," IEEE Transactions on Industry Applications, vol. 33, pp. 722-731, No. 3, (1997).

[2] J. Knockaert, J. Peuteman, J. Catrysse and R. Belmans, "Hidden reflection phenomena on inverter-fed induction motors", Proc. EPE 2005 Dresden (CD rom), (2005).

[3] J. Knockaert, J. Peuteman, J. Catrysse, R. Belmans, "Simulation of stair stepping on inverter-fed induction motors," International symposium on electromagnetic compatibility, Barcelona, Spain, Sept.4-8, pp. 230-234 (2006).

[4] E. Persson, "Transient effects in applications on PWM inverters to induction motors," IEEE Transactions on Industrial Applications, vol. 28, pp. 1095 - 1101, (1992).

[5] S. Van Haute, A. Malfait, R. Reekmans, and R. Belmans, "Losses, audible noise, and overvoltage in induction motor drives," Proc. IEEE PESC, pp. 585592, (1995).

[6] "Transmission line effects in PCB applications," Motorola Semiconductor AN, AN1051/D, (1990). 